Original Article

Journal of Epilepsy Research pISSN 2233-6249 / elSSN 2233-6257

Received September 30, 2016 Accepted October 24, 2016

Corresponding author: Sang Kun Lee Department of Neurology, Seoul National University Hospital, 101 Daehak-ro, Jongno-gu, Seoul 03080, Korea

Tel. $+82-2-2072-2923$

Fax. $+82-2-36727553$

E-mail; sangkun2923@gmail.com

\section{Focal Epileptogenic Lesions in Adult Patients with Epilepsy and Generalized Epileptiform Discharges}

\author{
Dong Wook Kim', Seo-Young Lee ${ }^{2}$, Sang Kun Lee ${ }^{3}$ \\ ${ }^{1}$ Department of Neurology, Konkuk University School of Medicine, Seoul; ${ }^{2}$ Department of Neurology, Kangwon \\ National University, Chuncheon; ${ }^{3}$ Department of Neurology, Seoul National University Hospital, Seoul, Korea
}

\begin{abstract}
Background and Purpose: There are reports of successful resective epilepsy surgery for pediatric patients with epilepsy and generalized epileptiform discharges when they had focal epileptogenic lesions identified by MRI. However, there is limited information regarding adult patients with epilepsy who have both generalized epileptiform discharges and focal epileptogenic lesions.

Methods: To investigate the incidence and characteristics of adult patients who have both generalized epileptiform discharges and potentially epileptogenic lesions, we retrospectively analyzed data of clinical features and results of EEG and MRI of all patients with adult-onset epilepsy in a tertiary referral hospital.

Results: While 1315 patients were classified as having partial seizures, 207 patients were classified as having generalized seizures. Five of 207 patients $(2.4 \%)$ with generalized seizures had potentially epileptogenic lesions. All the epileptogenic lesions were congenital or acquired during early life, such as focal cortical dysplasia, dysembryoplastic neuroepithelial tumor, and cerebromalacic change because of perinatal injury. Conclusions: The presence of epileptogenic lesions in adult patients with generalized epileptiform discharges may be an incidental finding, but it has been suggested that some adult-onset epilepsy with generalized epileptiform discharges may actually have focal onset, which may have significant clinical implications for the choice of appropriate treatment. (2016;6:77-80)
\end{abstract}

Key words: Generalized epileptiform discharge, Focal seizure, Secondary bilateral synchrony

\section{Introduction}

The current classification of epileptic seizures employs a dichotomy that divides the seizures into those with generalized and focal onsets. The differentiation between generalized and partial seizures is based on clinical semiology and EEG findings, which show that the seizures originate in bilaterally distributed networks or within networks limited to one hemisphere. Although this classification scheme has merits in that it provides the most essential information regarding the choice of appropriate treatment and quality of life of the patients, ${ }^{1}$ recent classification has abandoned the dichotomy of focal versus generalized in referring to syndromes, to separate the clinical manifestation from the underlying epileptogenic pathology that produces epileptic seizures. ${ }^{2}$

With the technical advances in neuroimaging and the wide application of epilepsy surgery to remove the epileptogenic lesion, em- phasis has been increasingly placed on the radiological localization of epileptogenic areas. ${ }^{3}$ The presence of a radiological lesion was regarded as a piece of evidence heavily weighted in favor of a zone of seizure origin that is more or less independent of clinical presentation and EEG findings. ${ }^{4-6}$ In pediatric patients, successful resective epilepsy surgery has been documented for the selected patients despite the presence of generalized epileptiform discharges, and the presence of MRI-identified focal epileptogenic lesions was used as the guidance in the decision of surgical treatment. ${ }^{7,8}$ However, there is limited information regarding the adult patients with epilepsy who have both generalized epileptiform discharges and focal epileptogenic lesions. We performed this study to investigate the incidence of focal epileptogenic lesions in adult patients with epilepsy and generalized epileptiform discharges. We also evaluated the clinical features and characteristics of radiological lesions in these patients. 


\section{Methods}

We retrospectively reviewed the epilepsy registry of consecutive epilepsy patients presenting at the Epilepsy Clinic in Konkuk University Hospital in Seoul between March 2008 and December 2015 with (1) confirmation of diagnosis of adult-onset ( $\geq 18$ years) epilepsy, (2) adequate evaluation with epilepsy protocol MRI and EEG, and (3) available follow-up for at least 12 months. All MRI images were evaluated visually during clinical work-up by at least two experienced neuroradiologists and the presence of generalized epileptiform discharge on EEG was reviewed by at least two epileptologists. Patients with no epileptiform discharge at the initial study underwent repeated EEG studies up to three times to increase the accuracy of diagnosis. Classification of epileptic seizures was based on the clinical semiology and the results of MRI and EEG, but the presence of generalized epileptiform discharge was considered essential for the diagnosis of generalized seizure. We carefully examined the EEGs showing generalized epileptiform discharges to exclude the possibility of focal seizure onset with the characteristics of secondary bilateral synchrony (SBS), including consistent 'lead-in' discharges from one hemisphere, presence of focal interictal discharges confined to one hemisphere, persistent voltage asymmetry of the discharge, lower frequency less than $2.5 \mathrm{~Hz}$, considerable morphological variability from complex to complex, and a single site of phase reversal in transverse bipolar montages.

\section{Results}

In our epilepsy registry, 1315 patients were classified as having partial seizures, while 207 patients were classified as having generalized seizures. Among the 207 patients with generalized seizures, five patients (2.4\%) had focal epileptogenic lesions. There were two men and three women, and the mean age of onset was 23 years (range from 18 to 28 years). The lesions were located in the mesial frontal area in two patients, basal frontal in one patient, hippocampus in one patient, and parietal area in one patient. Two patients with mesial frontal lesions complained of recurrent absence seizure with memory decline, and the other three patients experienced generalized tonic-clonic seizures only. Interestingly, MRI diagnosis of the epileptogenic lesions were congenital or acquired during early life, including focal cortical dysplasia, dysembryoplastic neuroepithelial tumor, and cerebromalacic change because of perinatal injury (Table 1 , Fig. 1). EEG showed generalized 3 to $4 \mathrm{~Hz}$ spike-wave complexes in all patients, and hyperventilation, photic stimulation, and sleep did not affect the generation of generalized spike-wave. During the medical follow-up, carbamazepine or oxcarbazepine was tried for three of the patients, and none of them experienced aggravation of the seizures (Table 1).

\section{Discussion}

Although the presence of generalized epileptiform discharge is a cornerstone of the diagnosis of generalized seizures and has been considered as a contraindication for epilepsy surgery, it has become clear that selected pediatric patients with a focal brain lesion may be favorable candidates for epilepsy surgery despite the presence of generalized epileptiform discharges. ${ }^{7.8}$ The pathologies of the epileptogenic lesions in these patients were congenital, perinatal, or acquired during infancy, and it was suggested that the generalized epileptiform discharge may be a manifestation of potentially reversible secondary epileptogenesis resulting from an interaction between the early lesion and the developing brain. ${ }^{8}$ Because none of our patients underwent surgical treatment, the coexistence of the generalized epileptiform discharges and the focal epileptogenic lesions in the same

Table 1. Clinical features of patients with adult-onset epilepsy who have both generalized epileptiform discharges and focal epileptogenic lesions

\begin{tabular}{|c|c|c|c|c|c|c|}
\hline No. & Sex & Onset age & Semiology & EEG & MRI diagnosis & Others \\
\hline 1 & $\mathrm{~F}$ & 18 & $\begin{array}{l}\text { Absence seizure } \\
\text { Rare GTCS }\end{array}$ & $4 \mathrm{~Hz} \mathrm{~S}-\mathrm{W}$ & Lt mesial frontal FCD & Memory complaint \\
\hline 2 & $\mathrm{~F}$ & 31 & Absence seizure only & $3.5 \mathrm{~Hz} \mathrm{~S}-\mathrm{W}$ & $\begin{array}{l}\text { Lt mesial frontal } \\
\text { dysembryoplastic } \\
\text { neuroepithelial tumor }\end{array}$ & $\begin{array}{l}\text { Memory complaint } \\
\text { Partial effect by carbamazepine }\end{array}$ \\
\hline 3 & $\mathrm{~F}$ & 28 & GTCS only & $4 \mathrm{~Hz} \mathrm{~S}-\mathrm{W}$ & $\begin{array}{l}\text { Lt parietal old trauma or } \\
\text { ischemia }\end{array}$ & Carbamazepine was effective \\
\hline 4 & M & 20 & GTCS only & $3 \mathrm{~Hz} \mathrm{~S}-\mathrm{W}$ & Lt hippocampus FCD & Mild mental retardation \\
\hline 5 & M & 18 & GTCS only & $3.5 \mathrm{~Hz} \mathrm{~S}-\mathrm{W}$ & Lt basal frontal old ischemia & Oxcarbazepine was effective \\
\hline
\end{tabular}

FCD, focal cortical dysplasia; GTCS, generalized tonic clonic seizure, S-W, spike and wave complex; Lt, left; Rt, right; F, female; M, male. 


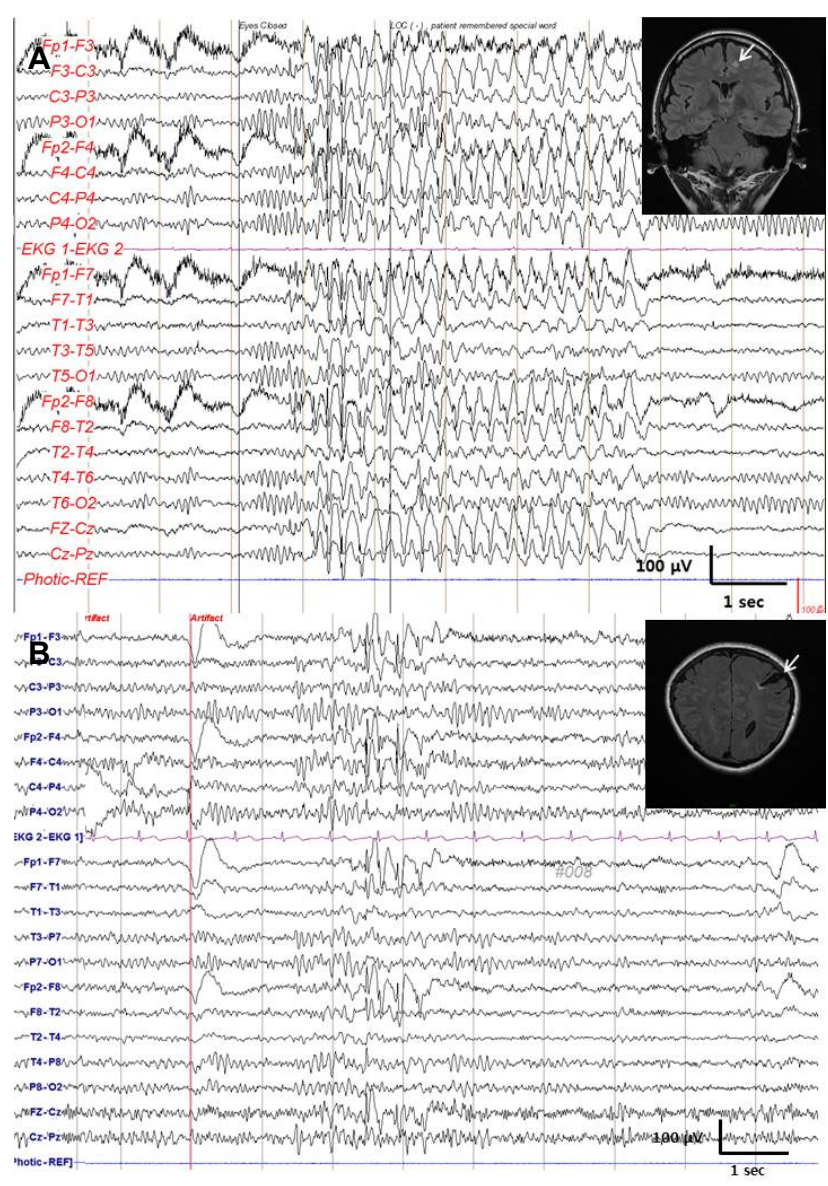

Figure 1. Illustrative cases. Patient No. 2: (A) MRI showing a left parasagittal frontal lesion and EEG showing a $4 \mathrm{~Hz}$ generalized spike and wave complex; (B) Patient No 4: MRI showing a left hippocampal lesion and EEG showing a $3 \mathrm{~Hz}$ generalized spike and wave complex.

patient may simply be an incidental finding. However, it is conceivable that the presence of generalized epileptiform discharge in our patients may also result from an interaction between the early lesion and the developing brain, because the MRI-identified lesions of our patients were also congenital or acquired during early life. However, there is still no explanation for why the patients began to experience clinical seizures during the adulthood with the congenital or early acquired lesions.

Patients with typical absence seizures show generalized spike-wave complexes at $3 \mathrm{~Hz}$. It has long been believed that the discharges of absence seizure originate from a deep-seated thalamic pacemaker to diffuse cortical areas, but there is increasing evidence that the cortex, especially the frontal area, may play an important role in the generation of generalized spike-wave discharges with a functionally intact thalamocortical network. ${ }^{9}$ In pediatric patients, there are several reports of absence seizure with generalized spike-wave discharges with focal epileptogenic lesions or preceding frontal spikes. ${ }^{4,10,11}$ Interestingly, the main clinical presentation of two patients with mesial frontal lesions was recurrent absence seizures with memory complaint, while the other patients experienced generalized tonic clonic seizures only.

SBS is characterized by bilateral synchronous discharges on EEG that can be shown to arise from a unilateral cortical focus. Although the differentiation of SBS from generalized epileptiform discharge is important in the evaluation of focal seizures, SBS are often indistinguishable from generalized epileptiform discharge on $\mathrm{EEG}$, so apparently generalized discharges in the EEG are not always associated with true generalized seizure onsets because a unilateral focal lesion can originate a discharge that spreads rapidly and synchronously across both hemispheres. ${ }^{12}$ The generation of generalized epileptiform discharge in our patients may share the same pathophysiology of SBS, and it can be suggested that the possibility of a focal epileptogenic lesion should be considered even in adult patients with generalized epileptiform discharges, especially when the patients have congenital and early acquired lesions on neuroimaging.

This study had several limitations. First, the study included a small number of patients over a limited period in a tertiary referral epilepsy clinic. Therefore, we may not be able to generalize our results to all patients with epilepsy. Second, only five patients were identified in our study, it was impossible to analyze the clinical features further in relation to the epileptogenic foci. Finally, because none of our patients underwent surgical treatment, we cannot confirm the causal relationship of focal epileptogenic lesions and generalized epileptiform discharges. However, we consider that our study has clinical implications, in that it shows the possibility that focal epileptogenic lesions should be considered despite the presence of apparent generalized epileptiform discharges.

\section{References}

1. Luders $H$, Acharya J, Baumgartner $C$, et al. A new epileptic seizure classification based exclusively on ictal semiology. Acta Neurol Scand 1999;99:137-41.

2. Berg AT, Berkovic SF, Brodie MJ, et al. Revised terminology and concepts for organization of seizures and epilepsies: report of the ILAE Commission on Classification and Terminology, 2005-2009. Epilepsia 2010;51:676-85.

3. Chauvel P, McGonigal A. Emergence of semiology in epileptic seizures. Epilepsy Behav 2014;38:94-103.

4. Holmes MD, Brown M, Tucker DM. Are "generalized" seizures truly 
generalized? Evidence of localized mesial frontal and frontopolar discharges in absence. Epilepsia 2004;45:1568-79.

5. Polkey CE. Clinical outcome of epilepsy surgery. Curr Opin Neurol 2004; 17:173-8.

6. Mortati KA, Arnedo V, Post N, Jimenez E, Grant AC. Sutton's law in epilepsy: because that is where the lesion is. Epilepsy Behav 2012;24:279-82.

7. Kramer U, Sue WC, Mikati MA. Focal features in West syndrome indicating candidacy for surgery. Pediatr Neurol 1997;16:213-7.

8. Wyllie E, Lachhwani DK, Gupta A, et al. Successful surgery for epilepsy due to early brain lesions despite generalized EEG findings.
Neurology 2007;69:389-97.

9. Meeren $H$, van Luijtelaar G, Lopes da Silva F, Coenen A. Evolving concepts on the pathophysiology of absence seizures: the cortical focus theory. Arch Neurol 2005;62:371-6.

10. Kakisaka Y, Alexopoulos AV, Gupta A, et al. Generalized 3-Hz spike-and-wave complexes emanating from focal epileptic activity in pediatric patients. Epilepsy Behav 2011;20:103-6.

11. Sofue $A$, Okumura $A$, Negoro $T$, et al. Absence seizures in patients with localization-related epilepsy. Brain Dev 2003;25:422-6.

12. Blume WT, Pillay N. Electrographic and clinical correlates of secondary bilateral synchrony. Epilepsia 1985;26:636-41. 